\title{
Development, Validation, and Testing of a Human Tissue Engineered Hypertrophic Scar Model
}

\author{
Leonarda J. van den Broek ${ }^{1}$, Frank B. Niessen ${ }^{2}$, Rik J. Scheper ${ }^{3}$, and Susan Gibbs ${ }^{1}$ \\ ${ }^{1}$ Department Dermatology, ${ }^{2}$ Plastic, Reconstructive and Hand Surgery and ${ }^{3}$ Pathology, MOVE Research Institute Amsterdam, \\ VU University Medical Center, Amsterdam, The Netherlands
}

\begin{abstract}
Summary
Adverse hypertrophic scars can form after healing of full-thickness skin wounds. Currently, reliable animal and in vitro models to identify and test novel scar reducing therapeutics are scarce. Here we describe the development and validation of a tissue-engineered human hypertrophic scar (HTscar) model based on reconstructed epidermis on a dermal matrix containing adipose derived mesenchymal stem cells (ASC). Although obtained from normal, healthy skin, ASC, in contrast to dermal mesenchymal cells, were found to facilitate HTscar formation. Quantifiable HTscar parameters were identified: contraction; thickness of dermis, collagen-1 secretion, epidermal outgrowth, epidermal thickness, and cytokine secretion (IL-6, CXCL8). The model was validated with therapeutics currently used for treating scars (5-fluorouracil, triamcinolon) and a therapeutic known to be unsuccessful in scar reduction (1,25-dihydroxyvitamin- $\left.D_{3}\right)$. Furthermore, it was shown that atorvastatin, but not retinoic-acid, may provide a suitable alternative for scar treatment. Each therapeutic selectively affected a different combination of parameters, suggesting combined therapy may be most beneficial. This animal-free hypertrophic scar model may provide an alternative model for mechanistic studies as well as a novel in vitro means to test anti-scar therapeutics, thereby reducing the use of animals.
\end{abstract}

Keywords: in vitro, skin equivalent, mesenchymal stem cell, scar, therapeutic

\begin{tabular}{|c|c|}
\hline \multicolumn{2}{|c|}{ Abbreviations } \\
\hline$\alpha$-SMA & alpha-smooth muscle actin \\
\hline ASC & adipose tissue derived mesenchymal stem cells \\
\hline CTGF & connective tissue growth factor \\
\hline $\mathrm{DE}$ & dermal equivalent \\
\hline DMSO & dimethylsulfoxide \\
\hline DSC & dermal derived mesenchymal stromal cells \\
\hline HTscar & hypertrophic scar \\
\hline $\mathrm{KC}$ & keratinocyte \\
\hline MSC & mesenchymal stem cells \\
\hline NTscar & normotrophic scar \\
\hline Nskin & normal skin \\
\hline P-DSC & $\begin{array}{l}\text { papillary dermal derived mesenchymal } \\
\text { stromal cells }\end{array}$ \\
\hline RA & all-trans-retinoic acid \\
\hline R-DSC & $\begin{array}{l}\text { reticular dermal derived mesenchymal } \\
\text { stromal cells }\end{array}$ \\
\hline SE & skin equivalent \\
\hline TC & triamcinolon \\
\hline $\mathrm{VitD}_{3}$ & 1,25-dihydroxy vitamin $\mathrm{D}_{3}$ \\
\hline $5 \mathrm{FU}$ & 5-fluorouracil \\
\hline
\end{tabular}

\section{Introduction}

Cuteanous wound healing is a natural, complex response to tissue injury and normally results in a scar. The most desirable scar is thin and flat and is mostly seen after superficial injury. This type is called a normotrophic scar (NTscar). Extensive trauma, deep burns, and sometimes even standard surgery, however, can result in wound closure with an adverse scar formation which is red, firm, raised, itchy, and painful. This adverse scar is known as a hypertrophic scar (HTscar) (Bayat et al., 2003). The quality of life of patients with HTscars can be severely affected due to loss of joint mobility, contractures, and disfigurements which lead to accompanying psychological problems (like depression and social avoidance) (Bayat et al., 2003).

HTscars occur more often after full-thickness wounding, where no viable dermis is left and adipose tissue is exposed. Therefore, the deeper the wound, the greater the possibility of HTscar formation (Deitch et al., 1983). The pathogenesis of HTscar formation in humans is not well understood, and although there are various treatment strategies, it is generally accepted that current strategies are still far from optimal (Atiyeh, 2007; Niessen et al., 1999). A major limitation in the progress of scar management is the lack of physiologically

Received March 7, 2012; accepted in revised form August 22, 2012 
relevant human models to explore the pathogenesis of HTscar formation and to test new therapeutics. Today, patients, animal models, and in vitro cell culture models are used to study skin scar formation. Patient studies are essential, but are limited by logistical and ethical problems. Common alternatives are animal studies. Despite the large number of studies describing pigs, mice, rabbits, and other animals as models to investigate hypertrophic scarring, the wound healing process in these species presents significant differences when compared with human scarring (Ramos et al., 2008). Pig skin most closely represents human skin and the red durac pig model has recently been validated, since these pigs develop HTscars similar to human HTscars in a number of ways (Zhu et al., 2007). However, extensive research with this model is limited due to the lack of pig specific biomarkers (such as those detected by monoclonal antibodies). Rabbit skin also shows some similarities to human scar formation, but the rabbit ear scar model (Morris et al., 1997) has similar restrictions to the pig model. Mouse models are used most extensively, even though mouse skin physiology poorly represents human skin and mice do not form adverse scars after wounding. Therefore, in order to humanize mouse models, studies have been described using $\mathrm{CXCR}^{-1-}$ mice (Yates et al., 2010) and transplanting human skin onto the backs of nude mice (Yang et al., 2007; Ramos et al., 2008). In addition to difficulties in interpreting results due to differences in skin physiology (and, in particular, scar formation), inflicting large, full-thickness trauma and burn wounds to animals has substantial ethical implications world-wide. In vitro cell culture models have been used to gain insight into different aspects of scar pathogenesis. Adipose derived mesenchymal cells, for example, have been described as having a number of similar characteristics to mesenchymal cells found within HTscar tissue, e.g., both are $\alpha$-SMA positive (El-Ghalbzouri et al., 2004; van den Bogaerdt et al., 2009; van der Veen et al., 2011; Wang et al., 2008). Additionally, a scratch assay has been described in which an increase in the single parameter Connective Tissue Growth Factor (CTGF) has been proposed for testing scar therapeutics (Moon et al., 2012). However, no attempts have been made so far to create a robust and physiologically relevant in vitro HTscar model for in vitro testing of therapeutics with multiple scar-forming parameters. With increasing pressure from the EU (Directive 2010/63/EU) for the replacement, reduction, and refinement of the use of animals models, there is an urgent need to develop a physiologically relevant in vitro human HTscar model to investigate the pathogenesis of HTscar formation. This, in turn, can facilitate identification and testing of new therapeutics leading to novel treatment strategies.

We have developed and validated a tissue engineered HTscar model consisting of a reconstructed epidermis on a dermal matrix populated with mesenchymal cells. We compared full-thickness skin equivalents (SE) constructed from mesenchymal stem cells isolated from the deep cutaneous adipose tissue (ASC) with SE constructed from more superficial mes- enchymal stromal cells found within the reticular dermis ( $\mathrm{R}$ DSC) and papillary dermis (P-DSC) in order to mimic HTscar formation, NTscar formation, and Nskin, respectively. We hypothesized that ASC in the exposed wound bed might most rapidly regenerate dermal tissue in order to close life threatening, deep cutaneous wounds at the cost of HTscar formation, whereas more superficial wounds are repaired from DSC within the flanking and underlying dermis, generally resulting in NTscar formation.

In order to develop, validate, and further test the HTscar model, a number of quantifiable parameters typical for HTscars were identified: 1) contraction, since HTscars are highly contractile (Ehrlich et al., 1994); 2) thickness of the dermis; 3) collagen-1 secretion, since more connective tissue is formed in HTscars than in NTscars (van der Veer et al., 2009a); 4) the degree of epithelialization, since it has been described that the extent of HTscar formation corresponds with delayed wound closure (Deitch et al., 1983); and 5) thickness of the regenerating epidermis, since it is known that HTscars have more epidermal cell layers than NTscars (Andriessen et al., 1998). In addition to the scar forming parameters, we assessed the secretion of two cytokines known to contribute to wound healing, IL-6 and CXCL8 (Broughton et al., 2006). The HTscar model was validated with therapeutics generally used in the clinic for scar treatment (5-fluorouracil and a triamcinolone (Kenacort $\left.{ }^{\circledR}-\mathrm{A} 40\right)$ ) (Mustoe et al., 2002; Wang et al., 2009) and a therapeutic known to be unsuccessful in scar reduction (1,25-dihydroxy vitamin $\mathrm{D}_{3}$ ) (van der Veer et al., 2009b). The HTscar model was further tested with two potential scar-reduction therapeutics (all-trans-retinoic acid and atorvastatin calcium salt trihydrate) (Aarons et al., 2007; Wang et al., 2009).

\section{Materials and methods}

\section{Normal skin and scar tissue}

Human adult skin samples were obtained from healthy individuals undergoing abdominal dermolipectomy or breast reduction surgery $(n=9$; age: $25-50$ years; sex: 8 x female, $1 \mathrm{x}$ male). Scar tissue samples were obtained from patients who underwent plastic surgery for scar excision (HTscar $n=8$; age: 25-55 years; sex: 7 x female, 1 x male; location: abdomen, breast, and flank; age of scar: $>1$ year and NTscar $n=7$; age: 15-60 years; sex: 6 x female, 1 x male; location: abdomen and breast; age of scar: $>1$ year). HTscars were defined as raised above skin level ( $>1 \mathrm{~mm})$ for at least 1 year and NTscars were defined as never raised above skin level. VU University Medical Center approved all the experiments described in this manuscript. The study was conducted according to the Declaration of Helsinki ${ }^{1}$.

\section{Cell isolation and culture of normal healthy skin}

Epidermal keratinocytes (KC) were isolated from healthy (non-scarred) human adult skin and cultured as described earlier (Waaijman et al., 2010). Keratinocytes were cultured until

1 http://www.wma.net/en/30publications/10policies/b3/ 
$80 \%$ confluence and then stored in the vapor phase of liquid nitrogen for later use.

Papillary dermal, reticular dermal, and adipose-derived mesenchymal cells were isolated by collagenase type II/dispase II treatment from healthy (non-scarred) human adult skin as previously described by Kroeze et al. (2009). In short, split thickness skin $(0.4 \mathrm{~mm})$ was removed using a dermatome (Acculan II, Braun, Tuttligen, Germany) to separate the papillary dermis from reticular dermis and adipose tissue (Schafer et al., 1985). The cells in the papillary dermis (upper layer) are further referred as P-DSC. From the remaining reticular dermis all adipose tissue was removed. Cells in the reticular dermis are further referred to as R-DSC. ASC were isolated in the same way as P-DSC and R-DSC. All mesenchymal cells were cultured under identical conditions and upon reaching $80 \%$ confluence were stored in the vapor phase of liquid nitrogen until required. Notably, within a single experiment, $\mathrm{KC}, \mathrm{P}-\mathrm{DSC}$, R-DSC, and ASC were all from the same donor. Cells at passage 3 were used to construct skin equivalents (SE), which consist of reconstructed epidermis on fibroblast populated dermal matrix, and dermal equivalents (DE) which are the same but lack an epidermis. Of note, P-DSC, and RDSC are the same cell population often referred to as dermal fibroblasts (Kroeze et al., 2009).

\section{Skin equivalents (SE) and dermal equivalents (DE)}

In this study we choose the sponge-like collagen-elastinmatrix (Matriderm ${ }^{\circledR}$; Dr. Suwelack Skin \& Health Care, Billerbeck, Germany) since it provides an initial scaffold for seeding the cells into but is then very easily remodeled by the cells within the matrix - thus enabling potential scar-like phenotypes to be formed. Mesenchymal cells (4 x 10 $0^{5}$ ) were seeded into the collagen-elastin-matrix $(2.2 \times 2.2 \mathrm{~cm})$ and cultured submerged for three weeks in culture medium containing DMEM (BioWhittaker, Verviers, Belgium)/Ham's F-12 (Invitrogen, GIBCO, Paisley, UK) (3:1), 2\% UltroSerG (UG) (BioSepra SA, Cergy-Saint-Christophe, France), 1\% penicillin/streptomycin (P/S) (Invitrogen, GIBCO, Paisley, UK), $5 \mu \mathrm{g} / \mathrm{ml}$ insulin, $50 \mu \mathrm{g} / \mathrm{ml}$ ascorbic acid, and $5 \mathrm{ng} / \mathrm{ml}$ epidermal growth factor (EGF). Unless otherwise stated, all culture additives were obtained from Sigma-Aldrich (St. Louis, MO, USA). Medium obtained after the last refreshment before keratinocytes were seeded onto the surface of DE was collected and is referred to as medium of DE. This conditioned DE medium was used for keratinocyte migration assays as described below. After 3 weeks of culturing, KC ( 5 x $10^{5}$ cells/culture) were seeded onto the surface of mesenchymal cell-populated matrixes. Then cultures were submerged for 4 days in DMEM/ Ham's F-12 (3:1), 1\% UG, 1\% P/S, $1 \mu \mathrm{M}$ hydrocortisone, 1 $\mu \mathrm{M}$ isoproterenol, $0.1 \mu \mathrm{M}$ insulin, and $1 \mathrm{ng} / \mathrm{ml} \mathrm{KGF}$. Hereafter, SE were cultured at the air-liquid interface in DMEM/ Ham's F-12 (3:1), 0.2\% UG, 1\% P/S, $1 \mu \mathrm{M}$ hydrocortisone, 1 $\mu \mathrm{M}$ isoproterenol, $0.1 \mu \mathrm{M}$ insulin, $10 \mu \mathrm{M}$ l-carnitine, $10 \mathrm{mM}$ l-serine, $1 \mu \mathrm{M}$ dl- $\alpha$-tocopherol acetate, and enriched with a lipid supplement containing $25 \mu \mathrm{M}$ palmitic acid, $15 \mu \mathrm{M}$ linoleic acid, $7 \mu \mathrm{M}$ arachidonic acid, and $24 \mu \mathrm{M}$ bovine serum albumin for another 10 days. SE were harvested after an entire culture period of 5 weeks for histological analysis and culture supernatants were collected for ELISA. The cultures received new culture medium twice a week.

\section{Application of therapeutics}

SE containing ASC were generated as described above. Therapeutics were added to the culture medium from the first medium renewal after starting the culture. The constructs were cultured with $10^{-7} \mathrm{M}$ all-trans-retinoic acid (Sigma-Aldrich, St. Louis, MO, USA), $10^{-8}$ M 5-Fluorouracil (Sigma-Aldrich, St. Louis, MO, USA), $10^{-7} \mathrm{M}$ Atorvastatin calcium salt trihydrate (Sigma-Aldrich, St. Louis, MO, USA) (all dissolved in $0.01 \%$ dimethylsulfoxide (DMSO), or $10^{-5}$ M Kenacort ${ }^{\circledR}-\mathrm{A} 40$ (Bristol-Myers Squibb B.V., Woerden, The Netherlands) (dissolved in $0.01 \%$ benzyl alcohol), or $10^{-8} \mathrm{M}$ 1,25-dihydroxy vitamin $\mathrm{D}_{3}$ (Sigma-Aldrich, St. Louis, MO, USA) (dissolved in $0.0095 \%$ ethanol). Corresponding vehicles were used as controls. The concentrations were determined from dose response studies on ASC monolayers. Concentrations were chosen at which ASC metabolic activity, corresponding to proliferation (2 days' exposure), was not inhibited in the MTT assay (see below).

\section{Histological and immunohistochemical analysis}

Paraffin embedded sections of normal tissue, scar tissue, and SE were used for morphological (haematoxylin and eosin staining) and immunohistochemical analysis (alpha-smooth muscle actin ( $\alpha$-SMA), clone 1A4; 1:200, Dako, Glostrup, Denmark) (Waaijman et al., 2010). The dermal thickness of SE was quantified from photos of H\&E stainings (Nikon Eclipse 80i Düsseldorf, Germany) taken at 200-fold magnification using NIS-Elements AR 2.10 software. The epidermal thickness was quantified by taking the mean of the number of living cell layers at 5 different regions within a single tissue section.

Measurement of matrix contraction and outgrowth of epidermis Matrix contraction and outgrowth of the epidermis were determined by taking photographs of the constructs at the first medium change and then again at the time of harvesting of the cultures. Photographs were taken with a Nikon Coolpix 5400 digital camera (Japan). The surface area of the constructs and the outgrowth of the epidermis outside of the original $1 \mathrm{~cm}$ diameter seeding area were determined using NIS-Elements AR 2.10 imaging software (Nikon).

\section{Keratinocyte migration}

Chemotactic migration of keratinocytes towards DE-conditioned medium (dose response of $0.3 \%, 3 \%$, and 30\%) with the aid of a modified Boyden well chamber technique using a 24-transwell system with $8 \mu \mathrm{m}$ was assessed and quantified as previously described (Kroeze et al., 2011).

\section{Cell proliferation}

A MTT assay was used to measure mitochondrial activity of ASC, which is representative of the viable number of cells (Mosmann, 1983). The assay was performed as described by the supplier (Sigma-Aldrich, St. Louis, MO, USA). 

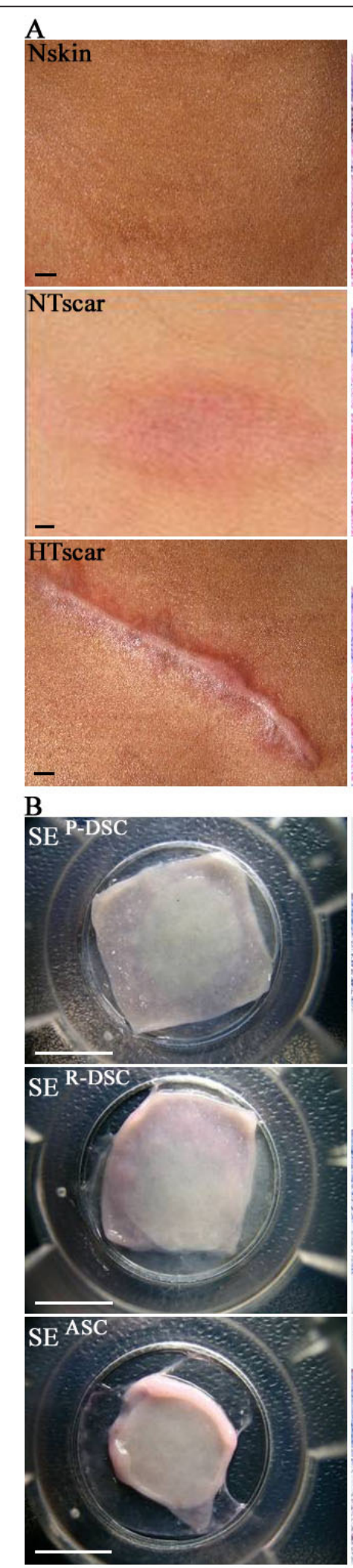
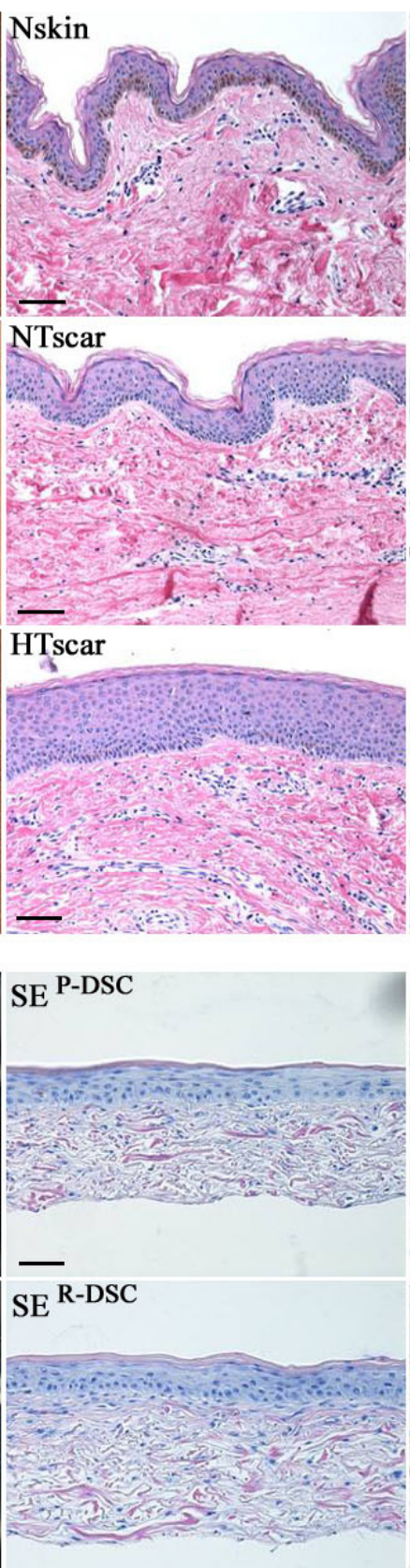

$\overline{S E}^{\mathrm{ASC}}$

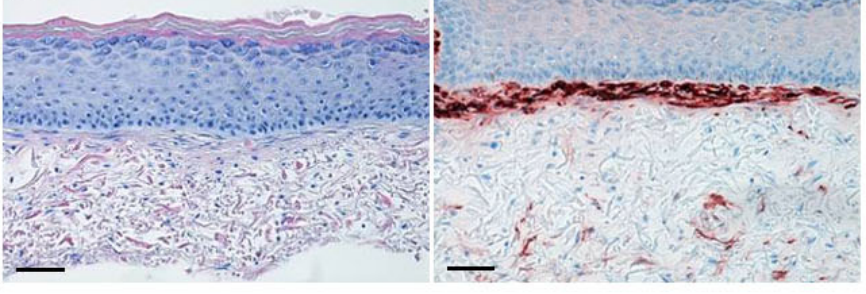

Fig. 1: Macroscopic and microscopic comparison of healthy skin with scar tissue and SE

A) Macroscopic overview, histological haematoxylin and eosin (H/E) staining, and immuno-histochemical a-SMA staining of human Nskin, NTscar, and HTscar tissue. B) Macroscopic overview, histological H/E staining, and immunohistochemical a-SMA staining of SE composed with P-DSC, R-DSC, and ASC. Bars macroscopic pictures $=1 \mathrm{~cm}$ and bars microscopic stainings $=100 \mu \mathrm{m}$. 
Relative proliferation of keratinocytes was determined by quantifying the amount of housekeeping enzyme lactate dehydrogenase $(\mathrm{LDH})$ released into the supernatant after $100 \%$ cell lysis with $0.1 \%$ Triton $\mathrm{X}-100$ as earlier described by Kroeze et al. (2011).

Enzyme-linked immunosorbent assay for cytokine production All reagents were used in accordance to the manufacturer's specifications. For collagen I quantification, commercially available ELISA antibodies and recombinant proteins obtained from Rockland (Gilbertsville, PA, USA) were used. For IL-6, commercially available paired ELISA antibodies and recombinant proteins obtained from R\&D System Inc. (Minneapolis, MN, USA) were used. For CXCL8 quantification, a Pelipair reagent set (CLB, Amsterdam, The Netherlands) was used.

\section{Statistical analysis}

At least three independent experiments were performed with each experiment being from a different donor and having an intra-experimental duplicate. Importantly, all experiments using KC, P-DSC, R-DSC, and ASC were donor-matched and performed in parallel. Difference in thickness and contraction of the matrix, outgrowth of the epidermis, number of epidermal cell layers, and collagen 1 secretion were compared between the different constructs using a repeated measures ANOVA test followed by Bonferroni's multiple comparison test. Difference in number of epidermal cell layers in native skin and scar tissues were compared using a one-way analysis of variance test, followed by Bonferroni's multiple comparison test. Differences in biomarker levels in the ASC model treated with therapeutic (compared to vehicle control) were assessed by paired t-test. Differences were considered significant when $\mathrm{P}<0.05$.

\section{Results}

\subsection{Qualitative macroscopic and microscopic comparison of native scars with the in vitro HTscar model}

In order to determine which characteristics are typical for a HTscar we first compared HTscar with native human NTscar and Nskin. Macroscopically, HTscar is more raised and red than NTscar and Nskin (Fig. 1A). Microscopically, HTscar has a thicker epidermis than NTscar and Nskin. Rete ridges are almost absent in HTscar and occur to a lesser extent in NTscar compared to Nskin (Ehrlich et al., 1994) (Fig. 1A). In order to identify the presence of myofibroblasts, which are thought to be mainly responsible for skin contraction after wounding, an $\alpha$-SMA staining was performed. In HTscars $\alpha$-SMA positive staining was not only observed around blood vessels but also in single cells in lower regions of the dermis. In contrast, both in NTscar and Nskin $\alpha$-SMA staining was mainly restricted to blood vessels (Fig. 1A).

Next we determined whether the SE constructed with either ASC, R-DSC or P-DSC showed typical macroscopic and microscopic characteristics of HTscar, NTscar and Nskin, respec-

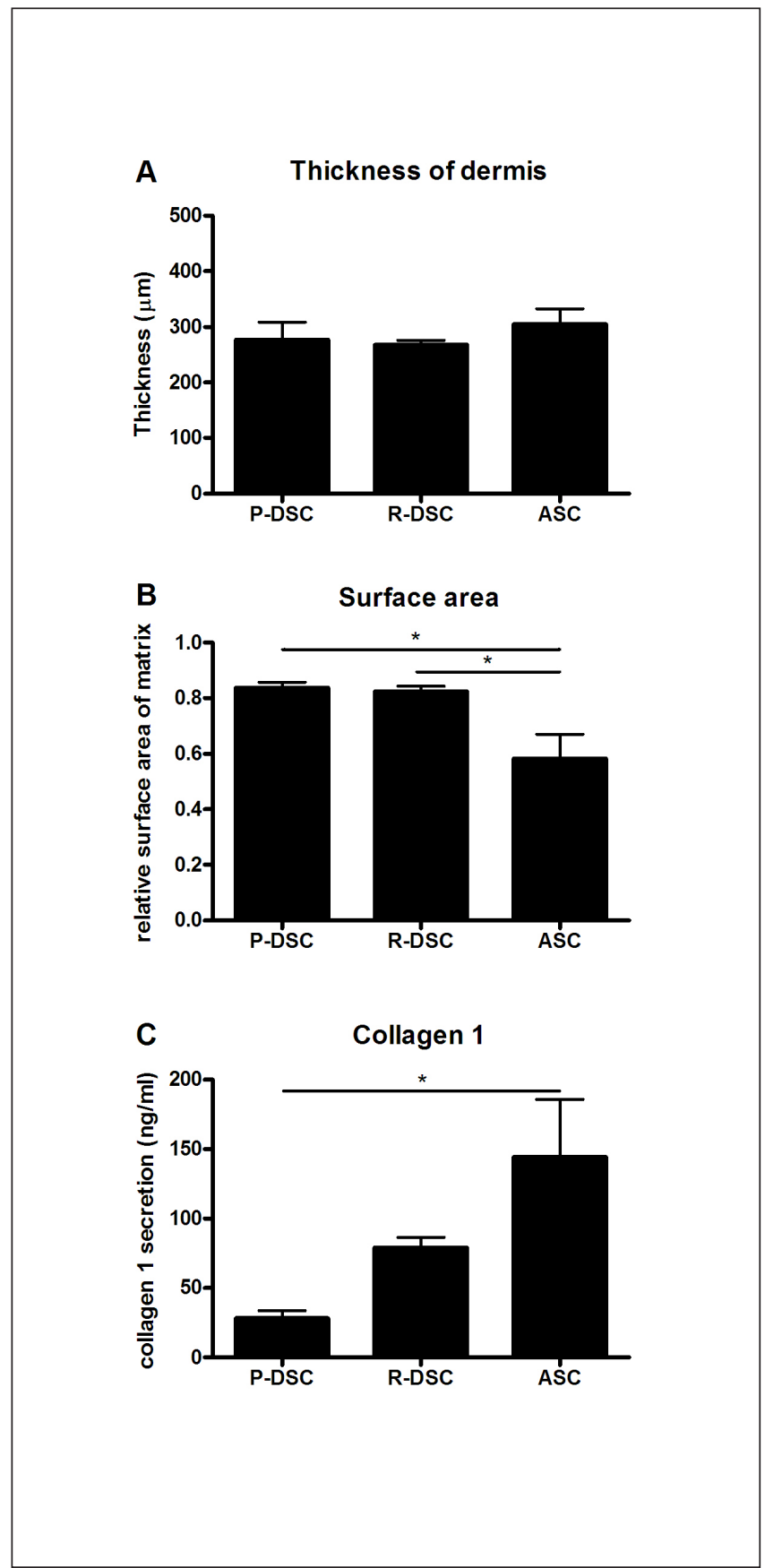

Fig. 2: Identification of dermal parameters for HTscar formation

A) Thickness of dermis of SE $(\mu \mathrm{m})$; B) relative matrix contraction of SE (surface area after 5 weeks of culture divided by surface at day 0); C) collagen 1 secretion into culture supernatants ( $\mathrm{ng} / \mathrm{ml}$ per equivalent per $24 \mathrm{~h}$ ). Experiments were performed with SE constructed from three different donors each in duplicate. Keratinocytes, P-DSC, R-DSC, and ASC were all from the same donor within a single experiment. Data are presented as the mean $(n=3 \pm S E M)$ thickness of dermis, contraction, or secretion of collagen 1. Statistically significant differences were calculated using a repeated measures ANOVA test followed by Bonferroni's multiple comparison test. *, $P<0.05 ;{ }^{* \star}, P<0.01$. 


\section{A Native tissue epidermal cell layers}

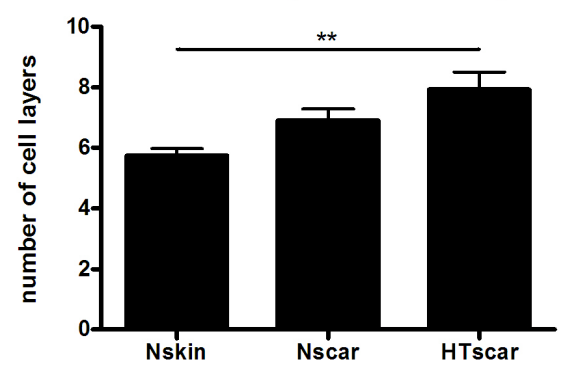

B

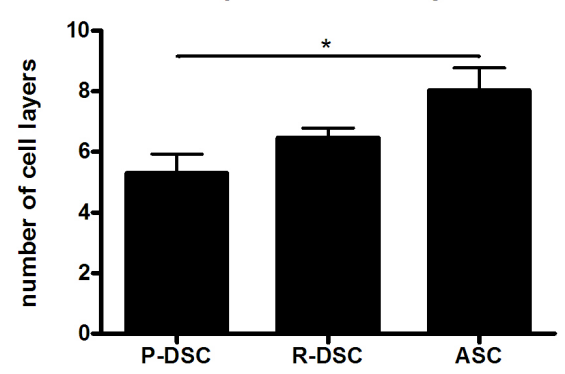

C

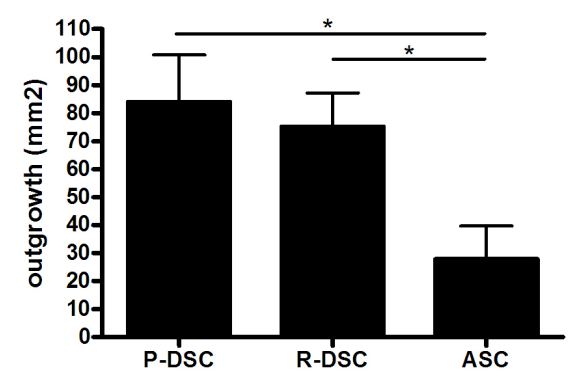

D

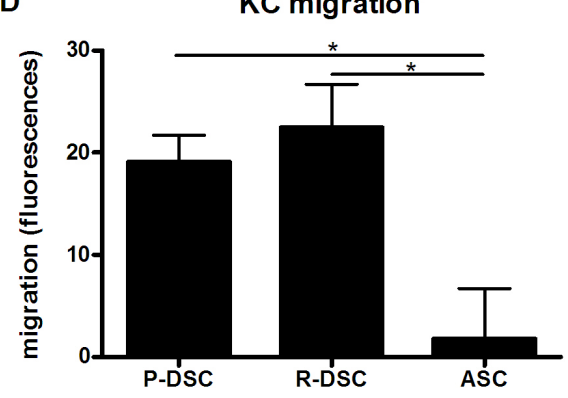

E KC proliferation

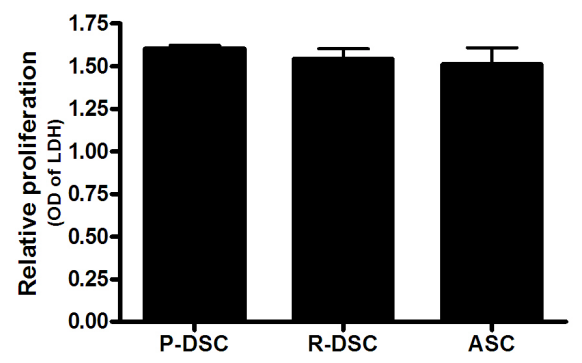

tively. Macroscopically, the $\mathrm{SE}^{\mathrm{ASC}}$ are more contractile than $\mathrm{SE}^{\mathrm{R}-\mathrm{DSC}}$ and $\mathrm{SE}^{\mathrm{P}-\mathrm{DSC}}$ (Fig. 1B). Similar to HTscar, microscopic examination of tissue sections showed that $\mathrm{SE}^{\mathrm{ASC}}$ had increased thickness of the epidermis. This was not observed with $\mathrm{SE}^{\mathrm{R}-\mathrm{DSC}}$ and $\mathrm{SE}^{\mathrm{P}-\mathrm{DSC}}$. There was increased $\alpha$-SMA staining in $\mathrm{SE}$, particularly in $\mathrm{SE}^{\mathrm{ASC}}$, where it is mainly located directly underneath the epidermis. The $\alpha$-SMA staining was much less extensive, and spread throughout the dermis in $\mathrm{SE}^{\mathrm{R}-\mathrm{DSC}}$ and $\mathrm{SE}^{\mathrm{P}-\mathrm{DSC}}$ (Fig. 1B).

Clearly, SE constructed with ASC-populated matrixes represent HTscars both macroscopically and microscopically, and have the potential for use in an in vitro HTscar model. In contrast, R-DSC and P-DSC visually represent NTscar and Nskin, respectively.

Before the HTscar model can be implemented, quantifiable and relevant parameters typical for HTscar need to be identified. Therefore, we next determined whether thickness of dermis, contraction, collagen 1 secretion, number of epidermal cell layers, and outgrowth of epidermis were suitable parameters. In addition, we determined whether the secretion of two cytokines related to wound healing, IL-6 and CXCL8, differed in the 3 different models.

\subsection{Identification of dermal parameters in the HTscar model}

In skin wound healing, the development of HTscar is characterized by an overproduction of extracellular matrix, increased contraction, and augmented $\alpha$-SMA expression compared to NTscar (Ehrlich et al., 1994). For this reason we first compared $\mathrm{SE}^{\mathrm{ASC}}$ with $\mathrm{SE}^{\mathrm{R}-\mathrm{DSC}}$ and $\mathrm{SE}^{\mathrm{P}-\mathrm{DSC}}$ with regards to thickness of the dermis, contraction, and collagen 1 secretion (Fig. 2).

The dermal thickness was not significantly different between the three SE (Fig. 2A). An increase in contraction is represented by a decrease in surface area of the SE and was

Fig. 3: Identification of epidermal parameters for HTscar formation

The epidermal thickness shown as the mean number of keratinocyte cell layers within the epidermis of $A$ ) native tissue biopsies and B) skin equivalents. C) The area of outgrowth of the epidermis outside of the original $1 \mathrm{~cm}$ diameter seeding area of SE $\left(\mathrm{mm}^{2}\right)$. D) Keratinocyte migration towards DE conditioned supernatant was assessed with a chemotactic transwell migration experiment. E) Relative proliferation of keratinocytes exposed to DE conditioned supernatant was determined by LDH assay. Experiments (triplicate) were performed from three different donors each in duplicate. Keratinocytes, P-DSC, R-DSC, and ASC were all from the same donor within a single experiment. Data are presented as the mean \pm SEM $(n=3)$. Statistically significant differences were calculated using a repeated measures ANOVA test followed by Bonferroni's multiple comparison test, except for the difference in number of epidermal cell layers in native skin and scar tissues, which were compared using one-way analysis of variance test, followed by Bonferroni's multiple comparison test.

${ }^{*}, P<0.05 ;{ }^{* *}, P<0.01$. 

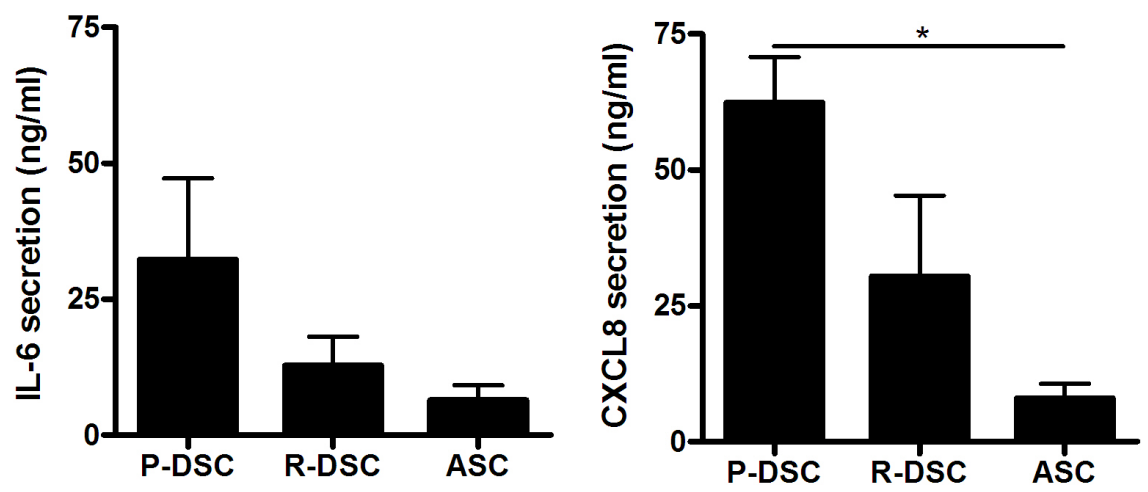

Fig. 4: Cytokine secretion

IL-6 and CXCL8 secretion by SE (ng/ml per equivalent per $24 \mathrm{~h}$ ). Data are presented as the mean \pm SEM secretion of IL-6 or CXCL8. Experiments (triplicate) were performed from three different donors, each in duplicate. Keratinocytes, P-DSC, R-DSC, and ASC were all from the same donor within a single experiment. Statistically significant differences between different $S E$ were calculated using repeated measures ANOVA test followed by Bonferroni's multiple comparison test. ${ }^{*}, P<0.05$.

observed for SE ${ }^{\mathrm{ASC}}$ compared to $\mathrm{SE}^{\mathrm{R}-\mathrm{DSC}}$ and $\mathrm{SE}^{\mathrm{P}-\mathrm{DSC}}$ (Fig. 2B). $\mathrm{SE}^{\mathrm{ASC}}$ secreted significantly more collagen 1 compared to $\mathrm{SE}^{\mathrm{P}-\mathrm{DSC}}$ (Fig. 2C).

From these results, contraction and collagen 1 secretion were identified as suitable dermal parameters for assessing HTscar formation in vitro using SE.

\subsection{Identification of epidermal parameters in the HTscar model}

It was observed that native HTscar had a thicker epidermis than NTscar and Nskin (Fig. 1A). This observation was confirmed by quantification of the number of epidermal cell layers: HTscar showed more epidermal cell layers $(7.9 \pm 1.6)$ than NTscars $(6.9 \pm 1.0)$ and Nskin $(5.8 \pm 0.6)$ (Fig. 3A). Next, we determined whether this increased epidermal thickness in native epidermis also occurred in the HTscar model. Indeed, $\mathrm{SE}^{\mathrm{ASC}}$ had an increased number of epidermal cell layers (8.00 $\pm 1.3)$ compared to $\mathrm{SE}^{\mathrm{R}-\mathrm{DSC}}(6.5 \pm 0.6)$ and $\mathrm{SE}^{\mathrm{P}-\mathrm{DSC}}(5.3 \pm 1.1)$ (Fig. 3B). Notably, all of these findings correlated very closely to native tissue and, in particular, HTscars had the same number of epidermal cell layers as $\mathrm{SE}^{\mathrm{ASC}}$.

Since the probability of HTscar formation is increased in wounds with delayed wound closure (Deitch et al., 1983), we next determined whether ASC were responsible for the delayed epidermal outgrowth compared to DSC. Indeed, SE ${ }^{\text {ASC }}$ had significant slower outgrowing epidermis compared with $\mathrm{SE}^{\mathrm{R}-\mathrm{DSC}}$ and SE ${ }^{\mathrm{P}-\mathrm{DSC}}$ (Fig. 3C). However, since the contraction is also greater in $\mathrm{SE}^{\mathrm{ASC}}$ compared with $\mathrm{SE}^{\mathrm{R}-\mathrm{DSC}}$ and $\mathrm{SE}^{\mathrm{P}-\mathrm{DSC}}$, it could not be entirely excluded from these findings that contraction confounded this result. To exclude the confounder, a chemotactic transwell migration experiment was performed with keratinocytes using conditioned supernatant derived from the three types of DE. The keratinocyte migration was reduced with supernatant derived from DE ${ }^{\mathrm{ASC}}$ compared with supernatants derived from $\mathrm{DE}^{\mathrm{R}-\mathrm{DSC}}$ and $\mathrm{DE}^{\mathrm{P}-\mathrm{DSC}}$ (Fig. 3D). The parallel proliferation experiment showed this decrease in migration was not due to changes in keratinocyte proliferation, indicating that ASC do indeed stimulate less epidermal migration than R-DSC and P-DSC.

From these results, the increase in number of epidermal cell layers and delayed outgrowth of epidermis were identified as suitable epidermal parameters for assessing HTscar formation in vitro using $\mathrm{SE}$.

\subsection{Cytokine IL-6 and CXCL8 secretion}

Most probably, already at the onset of wound healing, scar formation is initiated. Cytokines such as IL-6 and CXCL8 are reported to play a role in inflammation and granulation tissue formation during the wound healing process (Broughton et al., 2006). Therefore, the secretion of IL-6 and CXCL8 was assessed in culture supernatants derived from SE for their use as potential future novel scar parameters (Fig. 4).

The secretion of IL-6 was slightly lower (trend) when ASC were incorporated into SE than when P-DSC were used. The secretion of CXCL8 by the SE was significantly lower when ASC were incorporated into SE than when P-DSC were used.

From these results, decreased IL-6 and CXCL8 secretion were identified as a characteristic of $\mathrm{SE}^{\mathrm{ASC}}$.

\subsection{Validation and testing of the in vitro HTscar model with anti-scarring agents}

Clearly SE constructed from ASC populated matrixes not only visually represent HTscars, but also enabled quantifiable parameters to be identified, which are representative for HT- 


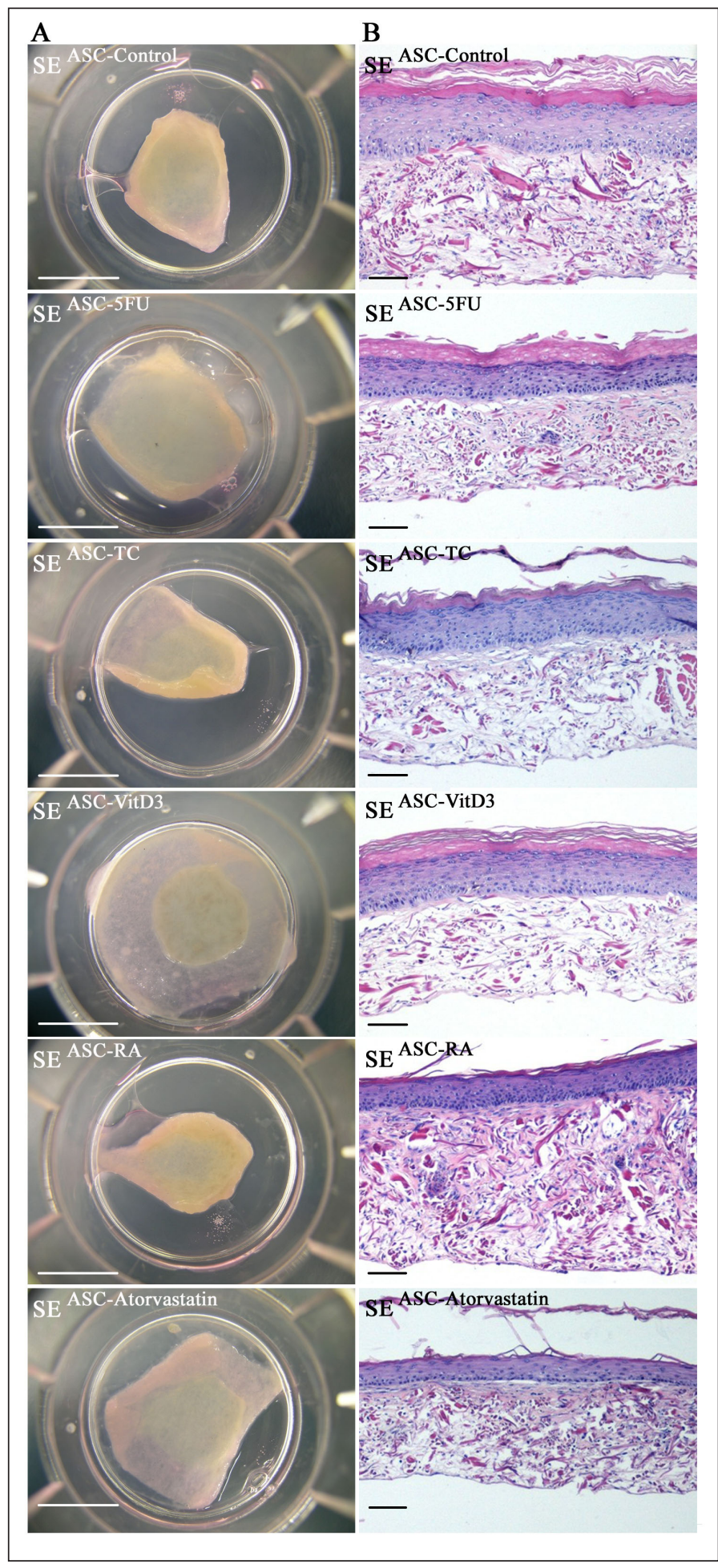

Fig. 5: Macroscopic and microscopic assessment of HTscar model cultured with therapeutics

A) Macroscopic overview (bars $=1 \mathrm{~cm}$ ) and B) Histological H/E staining (bars $=100 \mu \mathrm{m}$ ) of SE cultured without (control condition) and with therapeutics (5FU, TC, RA, VitD 3 , atorvastatin). For all therapeutics, vehicle controls were tested in parallel. The vehicle control conditions were similar to the control condition (no vehicle added), data not shown. scars. These were increases in 1) thickness of dermis; 2) contraction; 3) collagen 1 secretion; 4) number of epidermal cell layers and decreases in 5) degree of epithelialization. In addition, SE ${ }^{\mathrm{ASC}}$ showed reduced IL-6 secretion and reduced CXCL8 secretion.

The HTscar model was next validated by culturing with positive controls, i.e., two standard therapeutics (5-fluorouracil (5FU) and triamcinolon (TC)) which result in partial scar correction in patients, and a negative control, i.e., a therapeutic that is known to be ineffective in scar reduction (1,25-dihydroxy vitamin $\mathrm{D}_{3}\left(\mathrm{VitD}_{3}\right)$ (Tab. 1). Additionally, potential novel scar therapeutics (atorvastatin and all-trans-retinoic acid (RA)) were tested (Tab. 1). For all therapeutics, vehicle controls were tested in parallel. No significance was found between control condition (nothing added) and vehicle control conditions for the selected parameters. The results of this validation study are described below and summarized in Tab. 2, Fig. 5, and Fig. 6.

\section{5-fluorouracil (5FU): standard care (partially effective therapeutic)}

Supplementing $\mathrm{SE}^{\mathrm{ASC}}$ with $5 \mathrm{FU}$ led to reduced contraction (Fig. 5A, 6A) and reduced number of epidermal cell layers of $\mathrm{SE}$ compared to control $(6.3 \pm 0.8$ versus $7.8 \pm 0.9$ ) (Fig. 5B, 6B). Notably, $\mathrm{SE}^{\mathrm{ASC}}$ treated with $5 \mathrm{FU}$ had approximately the same number of epidermal cell layers as NTscars $(6.9 \pm 1.0)$ and SE ${ }^{\mathrm{R}-\mathrm{DSC}}(6.5 \pm 0.6)$ (Fig. 3A, B). No differences were found with regards to the other parameters (Fig. 6).

\section{Triamcinolon (TC): standard care (partially effective therapeutic)}

Supplementing TC reduced collagen 1 secretion of $\mathrm{SE}^{\mathrm{ASC}}$ (Fig. 6A). Also, the number of epidermal cell layers of SE ${ }^{\mathrm{ASC}}$ decreased after treating with TC compared to control ( $7.0 \pm 1.2$ versus $7.8 \pm 0.9)$ (Fig. 5B, 6B). No differences were found with regards to the other parameters.

\section{1,25-dihydroxy vitamin $D_{3}$ (VitD3): clinically non- effective therapeutic}

Supplementing $\mathrm{VitD}_{3}$ led to less contraction of $\mathrm{SE}^{\mathrm{ASC}}$ (Fig. 5A, 6A). The number of epidermal cell layers of $\mathrm{SE}^{\mathrm{A}-}$

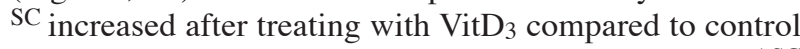
$(9.1 \pm 1.1$ versus $7.8 \pm 0.9)$ (Fig. $5 \mathrm{~B}, 6 \mathrm{~B})$. Notably, SE ${ }^{\mathrm{ASC}}$ treated with $\mathrm{VitD}_{3}(9.1 \pm 1.1)$ had even more epidermal cell layers than HTscars $(7.9 \pm 1.6)$ (Fig. 3A). The secretion of IL- 6 by SE ${ }^{\mathrm{ASC}}$ was even further reduced by adding $\mathrm{VitD}_{3}$ (Fig. 6C). No differences were found with regards to the other parameters.

\section{All-trans-retinoic acid (RA): potential novel scar therapeutic}

Supplementing potential novel scar therapeutic RA only partially normalized collagen 1 secretion of the HTscar model $\left(\mathrm{SE}^{\mathrm{ASC}}\right)$. No differences were found after supplementing $\mathrm{SE}^{\mathrm{ASC}}$ with RA with regards to the other param- 
Tab. 1: Potential of different therapeutics to treat HTscars

\begin{tabular}{|c|c|c|c|c|c|}
\hline & $5 F U$ & TC & VitD $_{3}$ & RA & Atorvastatin \\
\hline \multicolumn{6}{|l|}{ Clinical studies } \\
\hline Standard treatment & $\begin{array}{l}\text { Cancer / scar (Wang } \\
\text { et al., 2009) }\end{array}$ & $\begin{array}{l}\text { Scar (Wang et al., } \\
\text { 2009) }\end{array}$ & $\begin{array}{l}\text { Psoriasis (Ashcroft } \\
\text { et al., 2000) }\end{array}$ & $\begin{array}{l}\text { Leukemia (Wang } \\
\text { et al., 2009) }\end{array}$ & $\begin{array}{l}\text { Lowering } \\
\text { cholesterol (Aarons } \\
\text { et al., 2007) }\end{array}$ \\
\hline $\begin{array}{l}\text { Used in clinic for } \\
\text { scar treatment }\end{array}$ & $\begin{array}{l}\text { Yes (Wang et al., } \\
\text { 2009) }\end{array}$ & $\begin{array}{l}\text { Yes (Wang et al., } \\
\text { 2009) }\end{array}$ & No & No & No \\
\hline Response rate & $\begin{array}{l}50 \text { to } 86 \% \text { (Roques } \\
\text { and Teot, 2008) }\end{array}$ & $\begin{array}{l}50 \text { to } 100 \% \text { (Niessen } \\
\text { et al., 1999) }\end{array}$ & $\begin{array}{l}\text { No positive effect } \\
\text { ( } 30 \text { patients) (van } \\
\text { der Veer et al., 2009b) }\end{array}$ & - & - \\
\hline Recurrence rate & $\begin{array}{l}5-10 \% \text { (Roques } \\
\text { and Teot, 2008) }\end{array}$ & $\begin{array}{l}9 \text { to } 50 \% \text { (Niessen } \\
\text { et al., } 1999 \text { ) }\end{array}$ & - & - & - \\
\hline \multicolumn{6}{|l|}{ Preclinical studies } \\
\hline Neovascularization & - & $\downarrow$ (Wang et al., 2009) & - & $\begin{array}{l}\text { 个(Flynn and } \\
\text { Coleman, 2000) }\end{array}$ & - \\
\hline Animal experiments & - & - & - & - & $\begin{array}{l}\text { Yes, prevention } \\
\text { cardiac hypertrophy/ } \\
\text { and adhesions } \\
\text { (Aarons et al., 2007; } \\
\text { Senthil et al., 2005) }\end{array}$ \\
\hline Inflammation & - & $\begin{array}{l}\text { Anti-inflammatory } \\
\text { (Wang et al., 2009) }\end{array}$ & $\begin{array}{l}\text { Anti-inflammatory } \\
\text { (van der Veer et al., } \\
\text { 2009b) }\end{array}$ & $\begin{array}{l}\text { Regulator (Wang } \\
\text { et al., 2009) }\end{array}$ & $\begin{array}{l}\text { Anti-inflammatory } \\
\text { (Aarons et al., 2007) }\end{array}$ \\
\hline \multicolumn{6}{|l|}{ In vitro studies } \\
\hline $\begin{array}{l}\text { Fibroblast } \\
\text { proliferation }\end{array}$ & $\downarrow$ (Wang et al., 2009) & $\begin{array}{l}\downarrow(\text { Roques and } \\
\text { Teot, 2008) }\end{array}$ & $\begin{array}{l}\downarrow \text { (Greiling and } \\
\text { Thieroff-Ekerdt, 1996) }\end{array}$ & $\begin{array}{l}\downarrow(\text { Wang et al., } \\
2009)\end{array}$ & $\downarrow$ \\
\hline Collagen production & $\downarrow$ (Wang et al., 2009) & $\begin{array}{l}\downarrow \text { (Roques and } \\
\text { Teot, 2008) }\end{array}$ & $\begin{array}{l}\downarrow \text { (Greiling and } \\
\text { Thieroff-Ekerdt, 1996) }\end{array}$ & $\begin{array}{l}\downarrow \text { (Wang et al., } \\
2009)\end{array}$ & $\begin{array}{l}\downarrow \text { (Aarons et al., } \\
2007)\end{array}$ \\
\hline $\begin{array}{l}\text { Collagenase } \\
\text { production }\end{array}$ & - & - & - & $\begin{array}{l}\downarrow \text { (Wang et al., } \\
2009)\end{array}$ & - \\
\hline $\begin{array}{l}\text { Wound contraction / } \\
\text { myofibroblast }\end{array}$ & $\downarrow$ (Wang et al., 2009) & $\begin{array}{l}\downarrow(\text { Roques and } \\
\text { Teot, 2008) }\end{array}$ & $\begin{array}{l}\downarrow \text { (Greiling and } \\
\text { Thieroff-Ekerdt, 1996) }\end{array}$ & $\begin{array}{l}\downarrow \text { (Wang et al., } \\
2009)\end{array}$ & $\downarrow$ (Jiang et al., 2010) \\
\hline $\begin{array}{l}\text { Keratinocyte } \\
\text { proliferation }\end{array}$ & $\begin{array}{l}\downarrow \text { (Schwartz et al., } \\
\text { 1995) }\end{array}$ & $\begin{array}{l}\downarrow \text { (Roques and } \\
\text { Teot, 2008) }\end{array}$ & $\downarrow$ (Gibbs et al., 1996) & $\uparrow($ Gibbs et al., 1996) & - \\
\hline $\begin{array}{l}\text { Keratinocyte } \\
\text { differentiation }\end{array}$ & - & - & $\uparrow(G i b b s$ et al., 1996) & - & - \\
\hline $\begin{array}{l}\text { Future prospective } \\
\text { scar treatment }\end{array}$ & Yes & Yes & No & Potential & Potential \\
\hline
\end{tabular}

Tab. 2: HTscar parameters and cytokine secretion

\begin{tabular}{|c|c|c|c|c|c|c|c|}
\hline \multirow[b]{3}{*}{ Scar parameters } & \multirow[b]{3}{*}{ HT scar ${ }^{1}$} & \multirow[b]{3}{*}{ Model SEASC 2} & \multicolumn{5}{|c|}{ Therapeutics applied to in vitro HTscar model } \\
\hline & & & \multicolumn{2}{|c|}{ Standard care } & \multirow{2}{*}{$\frac{\text { Not effective }}{\text { VitD }_{3}}$} & \multicolumn{2}{|c|}{ Potential novel } \\
\hline & & & $5 F U$ & TC & & RA & Atorvastatin \\
\hline Thickness of dermis & $\uparrow$ & $=$ & $=$ & $=$ & $=$ & $=$ & $\downarrow^{*}$ \\
\hline Contraction & $\uparrow$ & $\uparrow$ & $\downarrow^{*}$ & $=$ & $\downarrow^{*}$ & $=$ & $=$ \\
\hline Collagen 1 secretion & $\uparrow$ & $\uparrow$ & $=$ & $\downarrow^{\star}$ & $=$ & * & $=$ \\
\hline Epidermal Thickness & $\uparrow$ & $\uparrow$ & $\downarrow^{*}$ & $\downarrow^{*}$ & $\uparrow^{*}$ & $=$ & $\downarrow^{\star}$ \\
\hline Outgrowth of epidermis & $\downarrow$ & $\downarrow$ & $=$ & $=$ & $=$ & $=$ & $=$ \\
\hline \multicolumn{8}{|l|}{ Cytokine secretion } \\
\hline IL-6 secretion & $?$ & $\downarrow$ & $=$ & $=$ & $\downarrow^{*}$ & $=$ & $=$ \\
\hline CXCL8 secretion & $?$ & $\downarrow$ & $=$ & $=$ & $=$ & $=$ & $\uparrow^{*}$ \\
\hline
\end{tabular}

${ }^{1}$ HTscar compared to NTscar; ${ }^{2}$ ASC model compared to model containing R-DSC and P-DSC; = comparable, $\uparrow$ increased, $\downarrow$ decreased compared to control condition, ? unknown. Statistically significant difference between in vitro HTscar model cultured with therapeutics compared to in vitro HTscar model cultured with corresponding vehicle controls ( ${ }^{*} \mathrm{p}<0.05$ and ${ }^{* *} \mathrm{p}<0.01$, paired t-test) 


\section{A Dermal parameters}
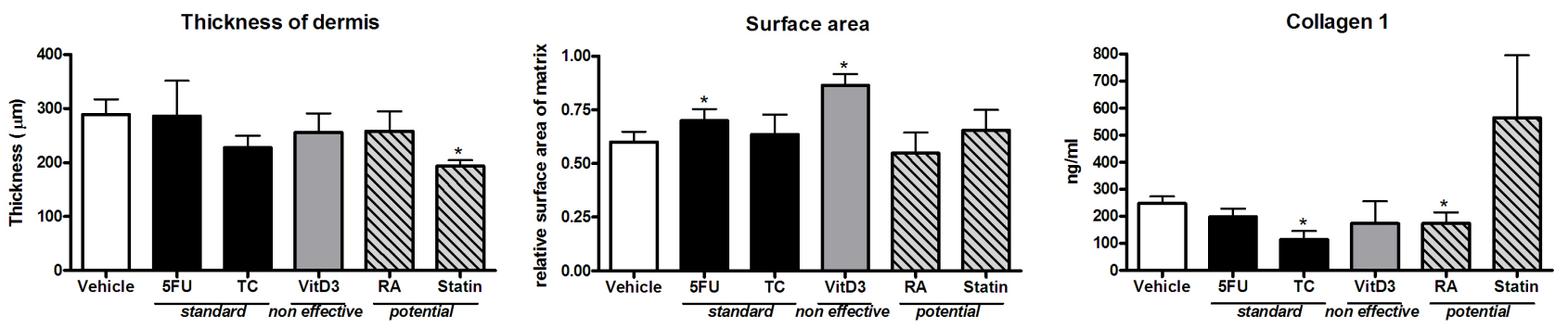

\section{B Epidermal parameters}
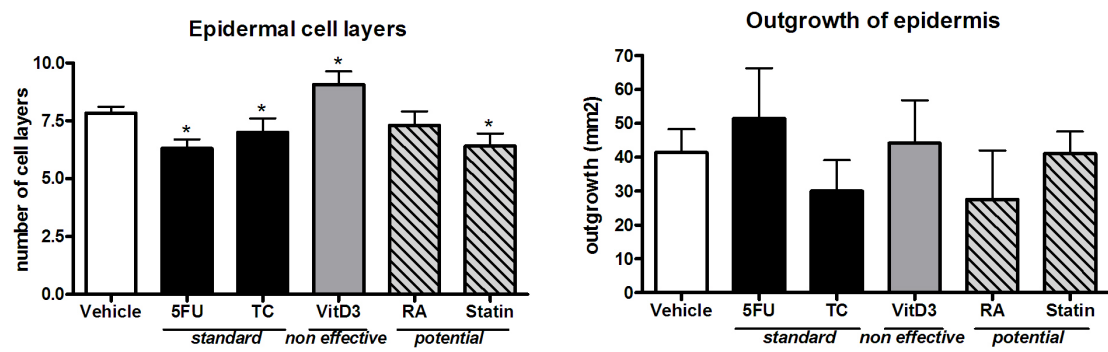

\section{Cytokine secretion}
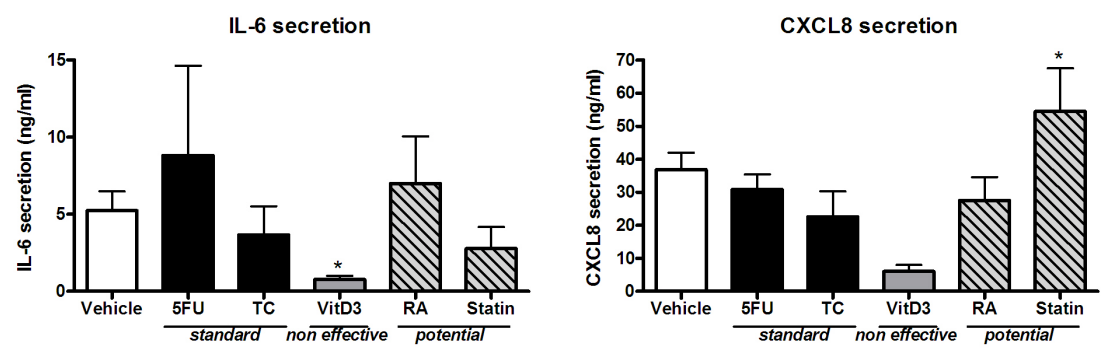

Fig. 6: Validation and testing of HTscar model with therapeutics

A) Dermal parameters: thickness of dermis $(\mu \mathrm{m})$, relative matrix contraction (surface after 5 weeks of culture divided by surface at day 0 ), and collagen 1 secretion ( $\mathrm{ng} / \mathrm{ml}$ per equivalent per $24 \mathrm{~h}$ ). B) Epidermal parameters: epidermal thickness (mean number of keratinocyte cell layers within the epidermis) and the area of outgrowth of epidermis outside of the original $1 \mathrm{~cm}$ diameter seeding area (mm2).

C) Cytokine secretion: IL- 6 and CXCL8 secretion ( $\mathrm{ng} / \mathrm{ml}$ per equivalent per $24 \mathrm{~h}$ ) by SE into culture supernatant was measured by ELISA. Vehicle = white bar; standard therapeutics = black bar; non effective therapeutics = grey bar; potential therapeutics = hatched bar.

Experiments (triplicate) were performed with SE from three different donors, each in duplicate. Keratinocytes and ASC were all from the same donor within a single experiment. Data are presented as the mean \pm SEM $(n=3)$. Statistically significance between the HTscar SE exposed to therapeutic and its corresponding vehicle was calculated using a paired t-test. For all therapeutics, vehicle controls were tested in parallel. No significance was found between control condition and vehicle control conditions for the selected parameters (data not shown). Therefore, all control conditions are grouped together in the white bar. The experiments were performed with three donors each in duplicate. ${ }^{*}, P<0.05$.

eters. These results indicate that RA was not an effective antiscar therapeutic in the HTscar model.

\section{Atorvastatin: potential novel scar therapeutic}

Supplementing $\mathrm{SE}^{\mathrm{ASC}}$ with atorvastatin reduced the thickness of the dermis (Fig. 5B, 6A).

The number of epidermal cell layers of $\mathrm{SE}^{\mathrm{ASC}}$ decreased after treating with atorvastatin compared to control $(6.4 \pm 1.0$ versus $7.8 \pm 0.9$ ) (Fig. 5B, 6B). Notably, $\mathrm{SE}^{\mathrm{ASC}}$ treated with atorvastatin had approximately the same number of epidermal cell layers as NTscars $(6.9 \pm 1.0)$ and SE ${ }^{\mathrm{R}-\mathrm{DSC}}(6.5 \pm 0.6)$ (Fig. $3 \mathrm{~A}, \mathrm{~B})$. The secretion of CXCL8 by $\mathrm{SE}^{\mathrm{ASC}}$ was increased by adding atorvastatin (Fig. 6C). No differences were found with regards to the other parameters. Atorvastatin was the only therapeutic tested which resulted in partial normalization of three parameters. 


\section{Discussion}

In this study we show that ASC and keratinocytes (both isolated from healthy, full-thickness human skin which is readily obtained as waste material after standard surgical procedures) may be used to establish an in vitro HTscar model to test anti-scarring therapeutics. The HTscar model had similar characteristics as HTscars and enabled relevant and quantifiable HTscar parameters to be identified and tested. Our first results, shown in this study, indicate that the in vitro HTscar model may be used to test potential anti-scar therapeutics. Testing with combinations of known therapeutics and novel therapeutics is now required to further investigate the value of the HTscar model with regards to replacement, reduction, and refinement of the use of animal models.

The first part of this study involved developing the HTscar model and selecting relevant and quantifiable HTscar parameters. We found that SE constructed with ASC visually represents HTscars. In contrast, incorporation of R-DSC and P-DSC, which are cells isolated from the more superficial layers of the skin, led to SE visually representing NTscar and Nskin, respectively. This observation is in line with the clinical observation that HTscars occur more often after the closure of full-thickness wounds (Deitch et al., 1983). Relevant and quantifiable parameters typical for HTscars that were identified in the HTscar model were contraction, collagen 1 secretion, outgrowth of epidermis, and epidermal thickness. Additionally, two cytokines typically involved in wound healing were assessed. The decrease in both IL-6 and CXCL8 secretion was characteristic for the HTscar model only and, therefore, it would now be interesting to determine whether HTscars in vivo also showed decreased expression of these cytokines. In literature, no consensus was reached whether IL-6 and CXCL8 are up- or down-regulated during HTscar formation (Zhou et al., 1997; Ricketts et al., 1996; Wang et al., 2011). The confusion may be due to size, location, and age of the studied scar samples. Although we did observe an increase in collagen 1 secretion, no increase in thickness of the dermis was observed in the presented HTscar model compared to SE composed with R-DSC and P-DSC. However, the thickness of the dermis was greater in DE when only ASC were incorporated into the matrix (without keratinocytes on top) than when R-DSC or P-DSC were used (data not shown). At present, the reason for this is unknown; this discrepancy between SE and DE, however, may be related to cultured keratinocytes being very active in secreting proteins which degrade the collagen matrix as it forms (Pilcher et al., 1998; Kahari and Saarialho-Kere, 1997).

Our results showed that dermal fibroblasts exhibited fewer hypertrophic scar characteristics than ASC, even though they have been reported to produce TGF $\beta 1$ and many cytokines involved in wound healing and scar formation (Nolte et al., 2008). This indicates, in line with results reported by others, that dermal fibroblasts are involved in normal wound healing, whereas ASC may be involved in adverse scar formation (van den Bogaerdt et al., 2009; van der Veen et al., 2011). Our finding that the SE ${ }^{\mathrm{ASC}}$ model secreted less IL-6 and CXCL8 may be of significance for the pathophysiology of scar formation and our model now provides an excellent means to investigate this further in parallel with in vivo patient derived-data. Of note, previously we have shown that ASC and dermal fibroblasts both display a mesenchymal stem cell phenotype (CD31 ${ }^{-}$, $\mathrm{CD}_{4}{ }^{+}, \mathrm{CD}_{45}{ }^{-}, \mathrm{CD}_{4} 4^{+}, \mathrm{CD} 90^{+}, \mathrm{CD} 105^{+}$, and $\left.\mathrm{CD} 166^{+}\right)$and show similar multi-lineage differentiation potential (Kroeze et al., 2009). These characteristics were more pronounced for ASC. This suggests that, possibly, potent mesenchymal stem cell capacity may correlate to poor scar quality and requires further investigation. Although our results are in line with the clinical observation that HTscars show increased $\alpha$-SMA compared to NTscar and Nskin, it was noticed that $\alpha$-SMA was strongly expressed directly below the basement membrane in the SE ${ }^{\text {ASC }}$ HTscar model. This indicates that cultured keratinocytes may secrete a factor which stimulates differentiation into $\alpha$-SMA positive cells. Interestingly, $\mathrm{DE}^{\mathrm{ASC}}$ showed very little $\alpha$-SMA expression, supporting this hypothesis (data not shown). Since the immunohistochemical staining of $\alpha$-SMA positive cells is difficult to quantify, this biomarker was not selected as a scar forming parameter.

The second aim of this study was to validate the HTscar model with two therapeutics regularly used in the clinic for scar treatment (5FU and TC) (Wang et al., 2009) and one therapeutic known to be unsuccessful in scar reduction $\left(\mathrm{VitD}_{3}\right)$ (van der Veer et al., 2009b). Supplementing the HTscar model with 5FU resulted in partial normalization of the contraction and the epidermal thickness. Interestingly, the other therapeutic, TC, resulted in partial normalization of a different pair of parameters: collagen 1 secretion and epidermal thickness. This finding indicates that combined therapy with 5FU and TC may have a better therapeutic effect than either single therapy. Indeed it has been shown in a clinical study (60 patients) that the combination of 5FU and TC does give a better response rate than either therapeutic alone (Asilian et al., 2006). Not all parameters (thickness of dermis, outgrowth of epidermis, IL6 secretion, and CXCL8 secretion) were favorably influenced by these two therapeutics. This result is in line with clinical results for 5FU and TC, since it is known that neither of these therapies can completely restore scar tissue to a normal skin phenotype in all patients (Tab. 1) (Niessen et al., 1999; Roques and Teot, 2008; Wang et al., 2009). Both standard therapeutics normalized only two parameters out of seven, indicating that for a therapeutic to be potentially effective, it should also partially normalize at least two parameters.

VitD3 was used as a negative control therapeutic in our study based on clinical evidence (van der Veer et al., 2009b). In line with the negative clinical results, we found an increased number of epidermal cell layers after adding VitD 3 . After adding VitD 3 , both IL-6 and CXCL8 were even further reduced. However, we also observed a decrease in contraction in SE which may be due to $\mathrm{VitD}_{3}$ inhibiting ASC proliferation, resulting in fewer cells in the matrix at time of harvesting. Indeed, FACScan flow cytometry analysis of $3 \mathrm{~mm}$ punch biopsies isolated from SE showed $48 \%$ less CD90+ cells within the dermis of $\mathrm{VitD}_{3}$ exposed $\mathrm{SE}$ compared to control 
vehicle exposed SE (data not shown). Despite the (thus far) reported clinical results, properly dosed $\mathrm{VitD}_{3}$ may possibly prove to be beneficial to the patient, since a decrease in the number of fibroblasts would result in fewer $\alpha$-SMA positive cells and less contraction. Therefore, further clinical studies are justified.

After testing the positive and negative controls, two therapeutics of unknown capacity to reduce HTscar characteristics (RA and atorvastatin) were tested. RA is an active metabolite of vitamin A and was included in this study since it decreases fibroblast proliferation and collagen production (Wang et al., 2009). However, our results indicate that RA may have limited value for scar treatment since it only partially normalized one HTscar parameter (reduction of collagen 1 secretion). Furthermore, this favorable effect may be counteracted by simultaneously decreasing collagen degradation (Wang et al., 2009). On the other hand, atorvastatin shows distinct therapeutic potential, since it was the only therapeutic to partially normalize three parameters (thickness of the dermis, epidermal cell layers, and CXCL8 secretion). These results are in line with the literature describing atorvastatin for preventing cardiac hypertrophy in rabbits and fibrotic adhesions in rats (Aarons et al., 2007; Senthil et al., 2005). Of note, this was the only therapeutic to reduce the thickness of the dermis a major parameter for a HTscar model. Since we showed in vitro that both $5 \mathrm{FU}$ and $\mathrm{TC}$ have partly complementary properties compared to atorvastatin, they may be potentially useful as combined therapies with atorvastatin. Our in vitro HTscar model will permit such pre-clinical investigations in the future.

The HTscar model constructed with ASC not only assesses HTscar reduction but also HTscar prevention, since therapeutics were applied to the culture medium from day 4 before SE were fully developed. This mimics early treatment after surgery. All selected parameters typical for HTscar were affected in SE by at least one of the tested therapeutics, with the exception of the outgrowth of the epidermis. This indicates that the model may be able to identify combinations of therapeutics that complement each other in correcting adverse scar formation.

As with all in vitro models, the HTscar model has a number of limitations that should be addressed. The main limitation is the lack of an immune component, since it is well known that infiltrating cells (e.g., macrophages, monocytes, etc.) influence wound healing (van der Veer et al., 2009a). Currently, the model is being further developed to include these immune cells in co-culture with the HTscar model. Also, neuro-endocrine signals (Ferreira et al., 2009) and an angiogenic component (van der Veer et al., 2011) have not yet been incorporated in this HTscar model. Also, extensive screening for more parameters, such as increased TGF $\beta 1$ (Campaner et al., 2006) or CTGF (Moon et al., 2012), might further improve the model and provide more insight into human HTscar formation. Another limitation is that only therapeutics that can be dissolved in the culture medium have been studied. It has yet to be determined whether similar results will be obtained if therapeutics are added topically to the stratum corneum of the SE. If this is the case, the model will also be suitable for testing waterinsoluble therapeutics in the form of creams and ointments. Also, the model will need further adapting if it is to test pressure and silicone dressings, both widely used in HTscar treatment (Tziotzios et al., 2012). The negative control therapeutic $\mathrm{VitD}_{3}$ gave one false positive result (contraction) and one correctly assessed result (increase in epidermal thickness) in addition to a decreased IL-6 and IL-8 secretion. However, it may be possible that the false positive result is a valid result and that the single clinical study described was performed under sub-optimal conditions with regards to $\mathrm{VitD}_{3}$ concentration. In general, though, a single false positive result can be minimized due to the assessment of multiple scar parameters.

In most academic research and during drug discovery studies, many animal experiments are used in the early phases to define and refine research questions and potential future applications. It is possible that these early stages of drug development can be replaced by our human in vitro HTscar model system, limiting animal experiments to the final in vivo confirmation and risk assessment phases. Generally, these final phases require maximally one-tenth of the total number of animals used (http://www.buzzle.com/articles/animal-testingstatistics.html).

In summary, we developed and validated an HTscar model using ASC and keratinocytes isolated from healthy skin and identified relevant and quantifiable parameters typical for HTscars. In line with the clinical experience, 5FU and TC only partially restored HTscar to normal skin phenotype. Each therapeutic selectively affected a different combination of parameters. These findings indicate that the in vitro model may be useful for selecting combinations of therapeutics with complementary properties. This will be a future area for investigation. Although the number of therapeutics tested in this initial study is small, our results indicate that this animal-free HTscar model may be used to test novel anti-scar therapeutics and may lead to the reduction of the use of animals in HTscar research.

\section{References}

Aarons, C. B., Cohen, P. A., Gower, A., et al. (2007). Statins (HMG-CoA reductase inhibitors) decrease postoperative adhesions by increasing peritoneal fibrinolytic activity. Ann Surg 245, 176-184.

Andriessen, M. P., Niessen, F. B., van de Kerkhof, P. C., et al. (1998). Hypertrophic scarring is associated with epidermal abnormalities: an immunohistochemical study.J Pathol 186, 192-200.

Ashcroft, D. M., Po, A. L., Williams, H. C., et al. (2000). Systematic review of comparative efficacy and tolerability of calcipotriol in treating chronic plaque psoriasis. BMJ 320 , 963-967.

Asilian, A., Darougheh, A., and Shariati, F. (2006). New combination of triamcinolone, 5-Fluorouracil, and pulsed-dye laser for treatment of keloid and hypertrophic scars. Der- 
matol Surg 32, 907-915.

Atiyeh, B. S. (2007). Nonsurgical management of hypertrophic scars: evidence-based therapies, standard practices, and emerging methods. Aesthetic Plast Surg 31, 468-492.

Bayat, A., McGrouther, D. A., and Ferguson, M. W. (2003). Skin scarring. BMJ 326, 88-92.

Broughton, G., Janis, J. E., and Attinger, C. E. (2006). The basic science of wound healing. Plast Reconstr Surg 117, 12S-34S.

Campaner, A. B., Ferreira, L. M., Gragnani, A., et al. (2006). Upregulation of TGF-beta1 expression may be necessary but is not sufficient for excessive scarring. $J$ Invest Dermatol 126, 1168-1176.

Deitch, E. A., Wheelahan, T. M., Rose, M. P., et al. (1983). Hypertrophic burn scars: analysis of variables. J Trauma 23, 895-898.

Ehrlich, H. P., Desmouliere, A., Diegelmann, R. F., et al. (1994). Morphological and immunochemical differences between keloid and hypertrophic scar. Am J Pathol 145, 105-113.

El-Ghalbzouri, A., van den Bogaerdt, A. J., Kempenaar, J., et al. (2004). Human adipose tissue-derived cells delay reepithelialization in comparison with skin fibroblasts in organotypic skin culture. Br J Dermatol 150, 444-454.

Ferreira, L. M., Gragnani, A., Furtado, F., et al. (2009). Control of the skin scarring response. An Acad Bras Cienc 81, 623-629.

Flynn, T. C. and Coleman, W. P. (2000). Topical revitalization of body skin. J Eur Acad Dermatol Venereol 14, 280-284.

Gibbs, S., Backendorf, C., and Ponec, M. (1996). Regulation of keratinocyte proliferation and differentiation by alltrans-retinoic acid, 9-cis-retinoic acid and 1,25-dihydroxy vitamin D3. Arch Dermatol Res 288, 729-738.

Greiling, D. and Thieroff-Ekerdt, R. (1996). 1alpha,25-dihydroxyvitamin D3 rapidly inhibits fibroblast-induced collagen gel contraction. J Invest Dermatol 106, 1236-1241.

Jiang, B. H., Tardif, J. C., Sauvageau, S., et al. (2010). Beneficial effects of atorvastatin on lung structural remodeling and function in ischemic heart failure. J Card Fail 16, 679688.

Kahari, V. M. and Saarialho-Kere, U. (1997). Matrix metalloproteinases in skin. Exp Dermatol 6, 199-213.

Kroeze, K. L., Jurgens, W. J., Doulabi, B. Z., et al. (2009). Chemokine-mediated migration of skin-derived stem cells: predominant role for CCL5/RANTES. J Invest Dermatol 129, 1569-1581.

Kroeze, K. L., Boink, M. A., Sampat-Sardjoepersad, S. C., et al. (2011). Autocrine Regulation of Re-Epithelialization After Wounding by Chemokine Receptors CCR1, CCR10, CXCR1, CXCR2, and CXCR3. J Invest Dermatol 132, 216225.

Moon, H., Yong, H., and Lee, A. R. (2012). Optimum scratch assay condition to evaluate connective tissue growth factor expression for anti-scar therapy. Arch Pharm Res 35, 383388.

Morris, D. E., Wu, L., Zhao, L. L., et al. (1997). Acute and chronic animal models for excessive dermal scarring: quantitative studies. Plast Reconstr Surg 100, 674-681.

Mosmann, T. (1983). Rapid colorimetric assay for cellular growth and survival: application to proliferation and cytotoxicity assays. J Immunol Methods 65, 55-63.

Mustoe, T. A., Cooter, R. D., Gold, M. H., et al. (2002). International clinical recommendations on scar management. Plast Reconstr Surg 110, 560-571.

Niessen, F. B., Spauwen, P. H., Schalkwijk, J., et al. (1999). On the nature of hypertrophic scars and keloids: a review. Plast Reconstr Surg 104, 1435-1458.

Nolte, S. V., Xu, W., Rennekampff, H. O., et al. (2008). Diversity of fibroblasts--a review on implications for skin tissue engineering. Cells Tissues Organs 187, 165-176.

Pilcher, B. K., Sudbeck, B. D., Dumin, J. A., et al. (1998). Collagenase-1 and collagen in epidermal repair. Arch Dermatol Res 290 Suppl, S37-S46.

Ramos, M. L., Gragnani, A., and Ferreira, L. M. (2008). Is there an ideal animal model to study hypertrophic scarring? J Burn Care Res 29, 363-368.

Ricketts, C. H., Martin, L., Faria, D. T., et al. (1996). Cytokine mRNA changes during the treatment of hypertrophic scars with silicone and nonsilicone gel dressings. Dermatol Surg 22, 955-959.

Roques, C. and Teot, L. (2008). The use of corticosteroids to treat keloids: a review. Int J Low Extrem Wounds 7, 137145 .

Schafer, I. A., Pandy, M., Ferguson, R., et al. (1985). Comparative observation of fibroblasts derived from the papillary and reticular dermis of infants and adults: growth kinetics, packing density at confluence and surface morphology. Mech Ageing Dev 31, 275-293.

Schwartz, P. M., Barnett, S. K., and Milstone, L. M. (1995). Keratinocytes differentiate in response to inhibitors of deoxyribonucleotide synthesis. J Dermatol Sci 9, 129-135.

Senthil, V., Chen, S. N., Tsybouleva, N., et al. (2005). Prevention of cardiac hypertrophy by atorvastatin in a transgenic rabbit model of human hypertrophic cardiomyopathy. Circ Res 97, 285-292.

Tziotzios, C., Profyris, C., and Sterling, J. (2012). Cutaneous scarring: Pathophysiology, molecular mechanisms, and scar reduction therapeutics Part II. Strategies to reduce scar formation after dermatologic procedures. J Am Acad Dermatol $66,13-24$.

van den Bogaerdt, A. J., van der Veen, V. C., van Zuijlen, P. P., et al. (2009). Collagen cross-linking by adipose-derived mesenchymal stromal cells and scar-derived mesenchymal cells: Are mesenchymal stromal cells involved in scar formation? Wound Repair Regen 17, 548-558.

van der Veen, V. C., Vlig, M., van Milligen, F. J., et al. (2011). Stem Cells in Burn Eschar. Cell Transplant 5, 933-942.

van der Veer, W. M., Bloemen, M. C., Ulrich, M. M., et al. (2009a). Potential cellular and molecular causes of hypertrophic scar formation. Burns 35, 15-29.

van der Veer, W. M., Jacobs, X. E., Waardenburg, I. E., et al. (2009b). Topical calcipotriol for preventive treatment of 
hypertrophic scars: a randomized, double-blind, placebocontrolled trial. Arch Dermatol 145, 1269-1275.

van der Veer, W. M., Niessen, F. B., Ferreira, J. A., et al. (2011). Time course of the angiogenic response during normotrophic and hypertrophic scar formation in humans. Wound Repair Regen 19, 292-301.

Waaijman, T., Breetveld, M., Ulrich, M., et al. (2010). Use of a collagen / elastin matrix as transport carrier system to transfer proliferating epidermal cells to human dermis in vitro. Cell Transplant 19, 1339-1348.

Wang, J., Dodd, C., Shankowsky, H. A., et al. (2008). Deep dermal fibroblasts contribute to hypertrophic scarring. Lab Invest 88, 1278-1290.

Wang, J., Hori, K., Ding, J., et al. (2011). Toll-like receptors expressed by dermal fibroblasts contribute to hypertrophic scarring. J Cell Physiol 226, 1265-1273.

Wang, X. Q., Liu, Y. K., Qing, C., et al. (2009). A review of the effectiveness of antimitotic drug injections for hypertrophic scars and keloids. Ann Plast Surg 63, 688-692.

Yang, D. Y., Li, S. R., Wu, J. L., et al. (2007). Establishment of a hypertrophic scar model by transplanting full-thickness human skin grafts onto the backs of nude mice. Plast Reconstr Surg 119, 104-109.

Yates, C. C., Krishna, P., Whaley, D., et al. (2010). Lack of CXC chemokine receptor 3 signaling leads to hypertrophic and hypercellular scarring. Am J Pathol 176, 1743-1755.
Zhou, L. J., Inoue, M., Ono, I., et al. (1997). The mode of action of prostaglandin (PG) I1 analog, SM-10906, on fibroblasts of hypertrophic scars is similar to PGE1 in its potential role of preventing scar formation. Exp Dermatol 6, 314-320.

Zhu, K. Q., Carrougher, G. J., Gibran, N. S., et al. (2007). Review of the female Duroc/Yorkshire pig model of human fibroproliferative scarring. Wound Repair Regen. 15, Suppl $1, \mathrm{~S} 32-\mathrm{S} 39$.

\section{Acknowledgements}

This study was financed by the Dutch Burns Foundation grant number 08.103.

\section{Correspondence to}

S. Gibbs, PhD

Department of Dermatology

VU University Medical Center

Room 2BR-028

De Boelelaan, 1081 HV Amsterdam

The Netherlands

Phone: +31204442815

Fax: +31 204442816

e-mail: S.Gibbs@vumc.nl 